\title{
Variabilidade espacial de atributos químicos do solo cultivado com soja sob plantio direto
}

\author{
Spatial variability of chemical attributes of soil cropped with soybean under no-tillage
}

\section{Flávio Carlos Dalchiavon ${ }^{1 *}$, André Ricardo Rodrigues ${ }^{2}$, Elizeu Souza de Lima ${ }^{3}$, Lenon Henrique Lovera ${ }^{3}$ e Rafael Montanari ${ }^{3}$}

Recebido em 06/03/2016 / Aceito em 15/12/2016

\section{RESUMO}

Durante o ano agrícola de 2012/2013, no município de Campo Novo do Parecis, MT, objetivou-se analisar a variabilidade espacial de atributos da soja e da fertilidade num Latossolo Vermelho distrófico típico cultivado sob plantio direto. Foi instalada a malha de amostragem para a coleta de dados numa área de 5,0 ha. Foram avaliadas a produtividade de grãos de soja, população de plantas, matéria orgânica, $\mathrm{P}, \mathrm{Ca}^{+2}, \mathrm{Mg}^{+2}, \mathrm{~K}^{+}, \mathrm{Al}^{+3}, \mathrm{H}^{+}+\mathrm{Al}^{+3}, \mathrm{pH}\left(\mathrm{CaCl}_{2}\right)$, soma de bases, capacidade de troca catiônica e saturação por bases. Efetuou-se a análise descritiva clássica, com auxílio do software estatístico SAS, análise de correlação e regressão com o Excel, sendo modelados semivariogramas para todos os atributos, obtendose as respectivas validações cruzadas e mapas de krigagens com o $\mathrm{GS}^{+}$7.0. A maior parte dos atributos pesquisados apresentou dependência espacial, sendo possível modelar seus respectivos semivariogramas, predominantemente do tipo gaussiano. Os alcances geoestatísticos utilizados para os atributos analisados não deverão ser inferiores a $43,8 \mathrm{~m}$, por serem a distância na qual os valores dos atributos estão relacionados entre si. $\mathrm{O}$ estudo da variabilidade dos atributos do solo bem como a adoção de técnicas que auxiliem no manejo do solo, como a geoestatística, é essencial ao adequado desenvolvimento vegetal.

PALAVRAS-CHAVE: agricultura de precisão, Glycine max., manejo e conservação do solo, semivariograma.

\section{ABSTRACT}

During the 2012/2013 agricultural year, in the city of Campo Novo do Parecis, MT, the study aimed to analyze the spatial variability of soybean and of the fertility attributes of Typic Tropudox under no-tillage. The sampling grid for data collect was installed in an area of 5.0 ha. The productivity of soybeans, plant population, organic matter, $\mathrm{P}, \mathrm{Ca}^{+2}, \mathrm{Mg}^{+2}, \mathrm{~K}^{+}, \mathrm{Al}^{+3}$, $\mathrm{H}^{+}+\mathrm{Al}^{+3}, \mathrm{pH}\left(\mathrm{CaCl}_{2}\right)$, sum bases, cation exchange capacity and base saturation were evaluated. A classic descriptive analysis was carried out with the help of the SAS statistical software, correlation and regression analysis with Excel and semivariograms were then modeled for all attributes, giving the respective cross-validations and kriging charts with the $\mathrm{GS}^{+} 7.0$ software. Most surveyed attributes presented spatial dependence, and modeled their semivariograms by predominantly Gaussian type. The geostatistical ranges used for the attributes analyzed should not be less than $43.8 \mathrm{~m}$, being the distance at which the attribute values are related to each other. The study of the variability of soil attributes and the adoption of techniques that help in the management of soil, such as geostatistics, is essential to proper plant development.

KEYWORDS: precision agriculture, Glycine max., soil fertility, management and soil conservation, semivariogram.

\section{INTRODUÇÃO}

O manejo do solo e da cultura (com destaque para a cultura da soja) faz parte da agricultura de precisão que envolve informações da variabilidade de planta e do solo visando o aumento da produtividade, otimização no uso dos recursos, fertilizantes e redução no impacto ambiental. Pesquisas têm demonstrado a importância da modelagem da variação espacial de atributos do solo que afetam a produtividade da cultura, com o objetivo de aperfeiçoar o aproveitamento

${ }^{1}$ Instituto Federal de Mato Grosso, Campo Novo do Parecis, MT, Brasil.

${ }^{2}$ Consultoria Agrodinâmica, Campo Novo do Parecis, MT, Brasil.

${ }^{3}$ Universidade Estadual Paulista, Ilha Solteira, SP, Brasil.

*Autor para correspondência < flavio.dalchiavon@cnp.ifmt.edu.br> 
de recursos, diminuir custos e auxiliar na gestão ambiental (DALCHIAVON et al. 2011a, SOUZA et al. 2014).

O conhecimento da variabilidade espacial dos atributos de solo e da cultura apresenta-se como ferramenta vantajosa para analisar a variabilidade de rendimento verificada e aperfeiçoar o manejo em áreas agrícolas por meio de um gerenciamento agrícola que leve em consideração informações pontuais de solo e de culturas (AMADO et al. 2009). O semivariograma é a principal ferramenta utilizada na geoestatística para a descrição da dependência espacial e consiste num grafico que associa distâncias com semivariâncias. Este tem a propriedade de medir o grau de dissimilaridade entre pares de medidas em razão da distância e orientação da linha entre dois locais de amostragem (SEIDEL \& OLIVEIRA 2014).

A aplicação de sistemas de manejo específico na agricultura exige informações precisas sobre a variação espacial dos atributos do solo e da cultura, que podem ser obtidas por meio dos mapas interpolados. Uma maior precisão das informações é adquirida quando se utiliza a intepolação por krigagem, pois estima valores para locais não estimados sem tendência e variação mínima, proporcionando uma estimativa mais precisa do atributo de interesse (SOUZA et al. 2014). Isso é possível por meio do estabelecimento de zonas específicas de manejo visando à conservação do solo (por exemplo, a aplicação de insumo com taxa variada), condições necessárias à agricultura de precisão (AP).

Visando estabelecer práticas sustentáveis de manejo do solo e das culturas é necessário o conhecimento dos atributos químicos do solo, com isso, o presente trabalho teve como objetivo analisar a variabilidade espacial de atributos químicos de um Latossolo Vermelho distrófico típico cultivado com soja sob plantio direto.

\section{MATERIAL E MÉTODOS}

A pesquisa foi implantada na área experimental do Instituto Federal de Educação, Ciência e Tecnologia de Mato Grosso, Campus Campo Novo do Parecis, num Latossolo Vermelho distrófico típico, de acordo com o Sistema Brasileiro de Classificação de Solos (EMBRAPA 2013), cujas coordenadas geográficas são: $13^{\circ} 40^{\prime} 31$ " de latitude Sul; 5753'31" de longitude Oeste e $572 \mathrm{~m}$ de altitude. Conforme preceitos de Köppen, o clima da região é o tropical úmido megatérmico (Aw), com temperaturas elevadas, chuva no verão e seca no inverno.

A cultura da soja (Glycine max L. Merril) foi semeada em 02 de dezembro de 2012 (variedade 98 Y30 RR), em sistema plantio direto. O espaçamento entre linhas foi de $0,45 \mathrm{~m}$, com uma população final de 16 plantas por metro na linha de semeadura, almejando uma população final de 260.000 plantas $\mathrm{ha}^{-1}$. A adubação de semeadura foi realizada conforme a análise química do solo $\left(400 \mathrm{~kg} \mathrm{ha}^{-1} \mathrm{da}\right.$ fórmula NPK 05-25-25), que revelou as seguintes características químicas: $\mathrm{pH}\left(\mathrm{H}_{2} \mathrm{O}\right)=5,6 ; \mathrm{MO}=24,2$ $\mathrm{g} \mathrm{dm}^{-3} ; \mathrm{P}$ (resina) $=1,0 \mathrm{mg} \mathrm{dm}^{-3} ; \mathrm{K}=21,5 \mathrm{mg} \mathrm{dm}^{-3} ; \mathrm{Ca}$ $=0,50 \mathrm{cmol}_{\mathrm{c}} \mathrm{dm}^{-3} ; \mathrm{Mg}=0,34 \mathrm{cmol}_{\mathrm{c}} \mathrm{dm}^{-3} ; \mathrm{H}+\mathrm{Al}=5,12$ $\mathrm{cmol}_{\mathrm{c}} \mathrm{dm}^{-3} ; \mathrm{S}=0,9 \mathrm{cmol}_{\mathrm{c}} \mathrm{dm}^{-3} \mathrm{e} \mathrm{T}^{\mathrm{c}}=6,0 \mathrm{cmol}_{\mathrm{c}} \mathrm{dm}^{-3}$.

Foram definidas as direções $\mathrm{x}$ e y do sistema de coordenadas cartesianas. Para tanto foi utilizada uma trena para a coleta dos pontos amostrais (cartesianos). A área pesquisada compreendeu 5,0 ha, onde foram distribuídos regularmente 100 pontos amostrais, de forma que o grid foi de 15 x $33 \mathrm{~m}$, nos eixos y e x, respectivamente. Desta forma, em média, cada ponto amostral ficou compreendido numa área de $495 \mathrm{~m}^{2}$. Os atributos do solo e da planta pesquisados foram todos individualmente coletados no entorno de cada ponto amostral da malha experimental. Em tal área, todos os 100 pontos foram alocados nas entrelinhas de semeadura, de forma que cada ponto de coleta de dados consistiu em quatro linhas de semeadura, sendo duas à esquerda e duas à direita de tal ponto. O comprimento de cada uma dessas quatro linhas de coleta (área amostral) foi de 3,00 m, totalizando a área útil de cada ponto amostral de $5,40 \mathrm{~m}^{2}(1,80 \times 3,00$ $\mathrm{m})$.

Os atributos pesquisados do solo foram: a) teores de matéria orgânica $(\mathrm{MO}), \mathrm{b}) \mathrm{P}, \mathrm{c}) \mathrm{Ca}^{+2}, \mathrm{~d}$ ) $\mathrm{Mg}^{+2}$, e) $\left.\mathrm{K}^{+}, \mathrm{f}\right) \mathrm{H}^{+}+\mathrm{Al}^{+3}, \mathrm{~g}$ ) valores de $\mathrm{pH}, \mathrm{h}$ ) soma de bases (S), i) capacidade de troca catiônica (T) e j) a saturação por bases (V\%), coletados na profundidade de 0-0,20 m. Já os atributos da planta foram: a) produtividade de grãos de soja (PRO) e b) população de plantas (POP). A colheita das plantas, assim como a coleta dos atributos do solo, foi realizada em 05 de março de 2013.

As amostras deformadas de solo, coletadas com trado de caneco com $0,10 \mathrm{~m}$ de diâmetro e 0,20 m de altura, foram utilizadas para determinação dos atributos químicos do solo, que foram realizadas no laboratório de fertilidade do solo da Faculdade de Engenharia de Ilha Solteira (FEIS), Universidade 
Estadual Paulista "Júlio de Mesquita Filho" (UNESP), conforme procedimentos definidos por RAIJ et al. (2001).

A população de plantas (POP) foi avaliada em campo contando-se, no momento da colheita, as plantas contidas nas duas linhas centrais de 3 metros em cada ponto amostral, estimando-se o resultado em número de plantas por hectare. A produtividade de grãos de soja (PRO), $\mathrm{kg} \mathrm{ha}^{-1}$, foi avaliada considerando quatro linhas de semeadura por três metros de comprimento, onde as plantas foram colhidas e, após secagem, submetidas à trilha manual, e em seguida a determinação da massa desses grãos, sendo os dados transformados em $\mathrm{kg} \mathrm{ha}^{-1}(13 \%$ b.u.), segundo metodologia apresentada em DALCHIAVON et al. (2011b).

Para cada atributo estudado, foi efetuado a análise descritiva clássica, com auxílio do software estatístico SAS (SCHLOTZHAVER \& LITTELL 1997), em que foram calculados a média, a mediana, valores mínimo e máximo, o desvio-padrão, o coeficiente de variação, o coeficiente de curtose, coeficiente de assimetria e construída a distribuição de frequências. Posteriormente, foi realizada a identificação dos outliers, efetuando a substituição dos seus valores pelo valor médio dos circunvizinhos contidos na malha experimental. Para testar a hipótese da normalidade, ou da lognormalidade, foi realizado o teste de SHAPIRO \& WILK (1965), a 5\% de probabilidade. Foi montada a matriz de correlação, objetivando efetuar as correlações lineares simples para as combinações, duas a duas, entre todos os atributos estudados. Assim, foram selecionados aqueles de maior correlação linear para a modelagem de regressões. Para a classificação do coeficiente de variação $(\mathrm{CV})$, adotou-se as seguintes classes e magnitudes: baixa $(\mathrm{CV} \leq 10 \%)$, média $(10 \%<\mathrm{CV} \geq$ $20 \%)$, alta $(20 \%<\mathrm{CV} \leq 0 \%)$ e muito alta $(\mathrm{CV}>30 \%)$ variabilidade (PIMENTEL-GOMES \& GARCIA 2002).

Isoladamente para cada atributo, foi analisada a sua dependência espacial pelo cálculo do semivariograma simples, desprezando a anisotropia local (modelo isotrópico), com base nos pressupostos de estacionaridade da hipótese intrínseca pelo uso do pacote Gamma Design Software 7.0 (GAMMA DESIGN SOFTWARE 2004). Os ajustes dos semivariogramas simples, em função de seus modelos, foram efetuados prioritariamente pela seleção inicial de: a) menor soma dos quadrados dos resíduos
(SQR); b) maior coeficiente de determinação $\left(\mathrm{r}^{2}\right)$ e c) maior avaliador da dependência espacial (ADE). A decisão final do modelo que representou o ajuste foi realizada pela validação cruzada, assim como para a definição do tamanho da vizinhança que proporcionou a melhor malha de krigagem, realizada por meio da krigagem em blocos. Para cada atributo, foram relacionados o efeito pepita $\left(\mathrm{C}_{\mathrm{o}}\right)$, o alcance $\left(A_{o}\right)$ e o patamar $\left(C_{o}+C\right)$. A análise do avaliador da dependência espacial (ADE) foi realizada conforme sugestões de CAMBARDELLA et al. (1994), modificada pelo GAMMA DESIGN SOFTWARE (2004), conforme a seguinte expressão:

$$
\mathrm{ADE}=\left[\mathrm{C} /\left(\mathrm{C}+\mathrm{C}_{\mathrm{o}}\right)\right] .100
$$

onde: $\mathrm{ADE}$ é o avaliador da dependência espacial; $\mathrm{C}$ é a variância estrutural; e $\mathrm{C}+\mathrm{C}_{\mathrm{o}}$ é o patamar. A interpretação proposta para o $\mathrm{ADE}$ foi conforme sugestão de DALCHIAVON \& CARVALHO (2012): a) $\mathrm{ADE}<20 \%=$ variável espacial de muito baixa dependência (MB); b) $20 \% \leq \mathrm{ADE}<40 \%=$ baixa dependência (BA); c) $40 \% \leq \mathrm{ADE}<60 \%=$ média dependência (ME); d) $60 \% \leq \mathrm{ADE}<80 \%=$ alta dependência (AL) e e) $80 \% \leq \mathrm{ADE}<100 \%=$ muito alta dependência (MA).

\section{RESULTADOS E DISCUSSÃO}

Observa-se que para os atributos da planta, PRO e POP, ocorreram CV's de 11,7 e 13,1\%, respectivamente. Para os atributos do solo, os CV's foram classificados como muito altos $(\mathrm{K}, \mathrm{Mg}$ e $\mathrm{Al})$, altos ( $\mathrm{P}, \mathrm{Ca}, \mathrm{H}+\mathrm{Al}, \mathrm{S}$ e $\mathrm{V} \%)$ e baixos $(\mathrm{MO}, \mathrm{pH}$ e $\mathrm{T})$, conforme a Tabela 1. Segundo CARVALHO et al. (2003), a variabilidade dos atributos químicos do solo é consequência de complexas interações dos processos de sua formação e de práticas de manejo do solo e da cultura, com impacto principalmente nas camadas superficiais do solo. Entretanto, no presente estudo, a variabilidade nos dados possivelmente esteve associada ao efeito de cultivos sucessivos em SPD, com a adubação realizada em linha.

De acordo com as classes de fertilidade do solo ( $\mathrm{P}, \mathrm{pH}, \mathrm{K}, \mathrm{Ca}, \mathrm{Mg}$ e V\%) propostas por RAIJ et al. (1997), do teor de matéria orgânica (ALVES et al. 2009) e do índice de saturação por alumínio (FONTES \& FONTES 1992), foi possível conhecer a distribuição dos referidos atributos no presente trabalho. Mediante tais classes, constatou-se que o P (com teor de 19,6 $\mathrm{mg} \mathrm{dm}^{-3}$ ) apresentou teor médio no solo (Tabela 1). Seu teor pode estar relacionado ao SPD pelo não 
Tabela 1 - Análise descritiva de atributos químicos em um Latossolo Vermelho distrófico típico. Campo Novo do Parecis (MT), 2013.

Table 1 - Descriptive analysis of chemical attributes of Typic Tropudox. Campo Novo do Parecis (MT), 2013.

\begin{tabular}{|c|c|c|c|c|c|c|c|c|c|c|}
\hline \multirow{3}{*}{ Atributo $^{(a)}$} & \multicolumn{10}{|c|}{ Medidas estatísticas descritivas } \\
\hline & \multirow[b]{2}{*}{ Média } & \multirow{2}{*}{ Mediana } & \multicolumn{2}{|c|}{ Valor } & \multirow{2}{*}{$\begin{array}{l}\text { Desvio } \\
\text { Padrão }\end{array}$} & \multicolumn{3}{|c|}{ Coeficiente } & \multicolumn{2}{|c|}{$\begin{array}{c}\text { Probabilidade } \\
\text { do teste }^{(b)}\end{array}$} \\
\hline & & & Mínimo & Máximo & & $\begin{array}{c}\text { Variação } \\
(\%)\end{array}$ & Curtose & Assimetria & $\operatorname{Pr}<\mathrm{w}$ & DF \\
\hline PRO (kg ha-1) & 3673 & 3695 & 2256 & 4583 & 430 & 11,7 & 0,218 & $-0,304$ & 0,562 & NO \\
\hline $\operatorname{POP}\left(\mathrm{pl} \mathrm{ha}{ }^{-1}\right)$ & 214370 & 214815 & 129630 & 281481 & 28075 & 13,1 & 0,089 & $-0,272$ & 0,538 & NO \\
\hline $\mathrm{P}\left(\mathrm{mg} \mathrm{dm}^{-3}\right)$ & 19,6 & 18,5 & 11,0 & 33,0 & 5,1 & 25,8 & $-0,548$ & 0,156 & 0,167 & $\mathrm{LN}$ \\
\hline $\mathrm{MO}\left(\mathrm{g} \mathrm{dm}^{-3}\right)$ & 24,5 & 25,0 & 20,0 & 29,0 & 2,0 & 8,2 & $-0,278$ & $-0,426$ & 0,001 & ND \\
\hline $\mathrm{pH}$ & 5,4 & 5,4 & 4,8 & 6,0 & 0,3 & 5,5 & $-0,478$ & 0,293 & 0,010 & ND \\
\hline $\mathrm{K}\left(\mathrm{mmol}_{\mathrm{c}} \mathrm{dm}^{-3}\right)$ & 1,9 & 1,7 & 0,4 & 3,7 & 0,8 & 41,5 & 0,458 & $-0,529$ & 0,050 & $\mathrm{LN}$ \\
\hline $\mathrm{Ca}\left(\mathrm{mmol}_{\mathrm{c}} \mathrm{dm}^{-3}\right)$ & 20,4 & 19,5 & 12,0 & 31,0 & 4,7 & 22,9 & $-0,333$ & $-0,080$ & 0,136 & $\mathrm{LN}$ \\
\hline $\mathrm{Mg}\left(\mathrm{mmol}_{\mathrm{c}} \mathrm{dm}^{-3}\right)$ & 14,9 & 13,0 & 6,0 & 25,0 & 4,4 & 31,4 & $-0,392$ & $-0,171$ & 0,216 & $\mathrm{LN}$ \\
\hline $\mathrm{H}+\mathrm{Al}\left(\mathrm{mmol}_{\mathrm{c}} \mathrm{dm}^{-3}\right)$ & 32,9 & 33,0 & 17,0 & 47,0 & 7,2 & 21,8 & $-0,498$ & 0,049 & 0,088 & NO \\
\hline $\mathrm{Al}\left(\mathrm{mmol}_{\mathrm{c}} \mathrm{dm}^{-3}\right)$ & 0,12 & 0 & 0 & 1 & 0,33 & 272,2 & 3,712 & 2,375 & $10^{-4}$ & ND \\
\hline $\mathrm{S}\left(\mathrm{mmol}_{\mathrm{c}} \mathrm{dm}^{-3}\right)$ & 36,3 & 34,8 & 19,6 & 60,3 & 9,2 & 25,4 & $-0,501$ & $-0,077$ & 0,377 & $\mathrm{LN}$ \\
\hline $\mathrm{T}\left(\mathrm{mmol}_{\mathrm{c}} \mathrm{dm}^{-3}\right)$ & 70,2 & 70,7 & 56,8 & 88,0 & 6,2 & 8,8 & 0,468 & 0,285 & 0,324 & NO \\
\hline V\% & 52,1 & 52,0 & 23,0 & 77,0 & 11,0 & 21,2 & $-0,365$ & 0,068 & 0,777 & NO \\
\hline
\end{tabular}

(a) PRO, POP, P, MO, pH, K, Ca, Mg, H+Al, Al, S, T e V\%1 são, respectivamente, a produtividade de grãos de soja, população de plantas, fósforo, matéria orgânica, potencial hidrogeniônico, potássio, cálcio, magnésio, acidez potencial, alumínio trocável, soma de bases, capacidade de troca catiônica e a saturação por bases; ${ }^{(b)} \mathrm{DF}=$ distribuição de frequência, sendo LN, ND e NO, respectivamente, do tipo lognormal, não determinada e normal.

revolvimento do solo, o que reduz o contato entre os colóides e o íon fosfato, amenizando as reações de adsorção, sobretudo pela adubação ter sido realizada na linha de semeadura, e ao P-orgânico, oriundo da decomposição dos restos de raízes remanescentes ao longo do perfil do solo, constituindo importante reserva de P-lábil para às plantas nas camadas mais profundas do solo.

Para os níveis de matéria orgânica no solo (MO), esta se apresentou na faixa de alto teor $(24,5 \mathrm{~g}$ $\mathrm{dm}^{-3}$ ), o que pode ser verificado na Tabela 1 . Uma das características marcantes do SPD é o acréscimo de MO, que decorre pelo fato da taxa de decomposição da palha mantida na superfície do solo ser menor do que se fosse incorporada. Em consequência, a alteração no teor de $\mathrm{MO}$, tanto em quantidade como em qualidade, tem implicações graduais nas alterações do $\mathrm{pH}$ (associada aos seus teores de cátions de reação básica e carbono orgânico solúvel) e na toxidez por Al, por meio de hidrólise ou complexação por ácidos orgânicos, conforme salientado por ZANÃO JÚNIOR et al. (2007).

$\mathrm{O}$ valor médio de $\mathrm{pH}(\mathrm{pH}=5,4)$, como pode ser verificado na Tabela 1, possibilitou um ambiente de acidez média ( $\mathrm{pH}$ entre 5,1 e 5,5), em virtude de que no ano de 2011 havia sido realizada a calagem na área, elevando-se a saturação por bases a $60 \%$. Em relação aos teores das bases trocáveis $\left(\mathrm{K}=1,9 \mathrm{mmol}_{\mathrm{c}}\right.$ $\left.\mathrm{dm}^{-3} ; \mathrm{Ca}=20,4 \mathrm{mmol}_{\mathrm{c}} \mathrm{dm}^{-3} ; \mathrm{Mg}=14,9 \mathrm{mmol}_{\mathrm{c}} \mathrm{dm}^{-3}\right) \mathrm{e}$ da $\mathrm{V} \%(\mathrm{~V} \%=52,1)$, estes classificaram o solo como de média ( $\mathrm{K}$ e $\mathrm{V} \%$ ) e alta fertilidades ( $\mathrm{Ca}$ e $\mathrm{Mg}$ ). Os teores de bases trocáveis bem como de $\mathrm{pH}$ e da $\mathrm{T}$ e V\% demonstraram o potencial do solo pesquisado para o cultivo da cultura da soja, o que pode ser verificada 
pela PRO (3673 $\left.\mathrm{kg} \mathrm{ha}^{-1}\right)$ e pela POP (214370 plantas ha $^{-1}$ ) obtidas no presente estudo. Em relação à PRO, observa-se que foi $31 \%$ superior a PRO média obtida $\left(2800 \mathrm{~kg} \mathrm{ha}^{-1}\right)$ no Brasil no mesmo ano (CONAB 2014).

Na Tabela 2 está apresentada a matriz de correlação linear de Pearson entre atributos de planta (PRO e POP) e atributos químicos do solo (P, MO, $\mathrm{pH}, \mathrm{K}, \mathrm{Ca}, \mathrm{Mg}, \mathrm{H}+\mathrm{Al}, \mathrm{Al}, \mathrm{S}, \mathrm{T}$ e V\%). Dentre as correlações entre a PRO e POP e os atributos do solo, a que apresentou significância de interesse no âmbito do manejo do solo foi o par PRO x P $\left(\mathrm{r}=0,215^{*}\right)$. Esta correlação direta indica que com o aumento do teor de $\mathrm{P}$ no solo haverá também o aumento da produtividade da soja, isso em virtude da importância que o $\mathrm{P}$ possui no solo e na planta, sendo um dos principais elementos relacionados ao fornecimento de energia à planta. Correlação positiva entre os teores de $\mathrm{P}$ no solo e a produtividade vegetal também foram evidenciadas por CAIONE et al. (2012) e COSTA et al. (2014), portanto, ratificaram a importância do correto manejo deste importante nutriente no solo.

Neste contexto, a equação de regressão obtida a partir de tais atributos está apresentada na Figura 1. Foi verificado que a PRO apresentou uma variação quadrática mediante ao aumento dos teores de $\mathrm{P}$ no solo, diferindo dos modelos lineares apresentados por COSTA et al. (2014) e MONTANARI et al. (2014). O ponto de máximo da equação ficou estabelecido em $24,6 \mathrm{mg} \mathrm{dm}^{-3}$ de $\mathrm{P}$, ou seja, a máxima produtividade agronômica $\left(3816,30 \mathrm{~kg} \mathrm{ha}^{-1}\right)$ da cultura foi obtida por ocasião do mencionado valor de $\mathrm{P}$ no solo, não sendo verdadeiro incrementos de produtividade da cultura a partir de valores de $\mathrm{P}$ no solo superiores a 24,6 $\mathrm{mg} \mathrm{dm}^{-}$ ${ }^{3}$, limitada pela inflexão da parábola. Por outro lado, considerando o valor médio de $\mathrm{P}$ no solo $\left(19,6 \mathrm{mg} \mathrm{dm}^{-}\right.$ ${ }^{3}$ ) apresentado na Tabela 1 , foi possível constatar que a PRO obtida foi de $3746,42 \mathrm{~kg} \mathrm{ha}^{-1}$ para este momento, valor este muito próximo da real produtividade obtida neste estudo, o que atesta a qualidade da regressão obtida.

Os dados não apresentaram distribuições simétricas, contudo, os coeficientes de assimetria e curtose ficaram próximos de zero, bem como valores médios e medianos próximos para a maioria dos atributos, o que permite que lhes sejam aplicados tratamentos geoestatísticos, segundo constatação mencionada por CHAVES \& FARIAS (2009) e por meio desta técnica possibilita ocorrer a avaliação da dependência espacial dos atributos.

Tabela 2 - Matriz de correlação linear simples entre atributos de plantas da soja e atributos químicos de um Latossolo Vermelho distrófico típico. Campo Novo do Parecis (MT), 2013.

Table 2 - Simple linear correlation matrix between the soybean plant attributes and chemical attributes of Typic Tropudox. Campo Novo do Parecis (MT), 2013.

\begin{tabular}{|c|c|c|c|c|c|c|c|c|c|c|c|c|}
\hline \multirow{2}{*}{ Atributos $^{(a)}$} & \multicolumn{12}{|c|}{ Coeficientes de correlação $0^{(b)}$} \\
\hline & PRO & POP & $P$ & $\mathrm{MO}$ & $\mathrm{pH}$ & $\mathrm{K}$ & $\mathrm{Ca}$ & $\mathrm{Mg}$ & $\mathrm{H}+\mathrm{Al}$ & $\mathrm{Al}$ & $\mathrm{S}$ & $\mathrm{T}$ \\
\hline POP & $0,284 * *$ & & & & & & & & & & & \\
\hline $\mathrm{P}$ & $0,215^{*}$ & 0,046 & & & & & & & & & & \\
\hline MO & 0,158 & $-0,027$ & $0,250^{*}$ & & & & & & & & & \\
\hline $\mathrm{pH}$ & 0,017 & $-0,063$ & $0,332 * *$ & 0,117 & & & & & & & & \\
\hline K & 0,033 & $-0,041$ & $0,357^{* *}$ & 0,165 & $0,312 * *$ & & & & & & & \\
\hline $\mathrm{Ca}$ & 0,129 & $-0,035$ & $0,499 * *$ & $0,324 * *$ & $0,788 * *$ & $0,375^{* *}$ & & & & & & \\
\hline $\mathrm{Mg}$ & 0,050 & $-0,044$ & $0,359 * *$ & $0,275^{* *}$ & $0,873 * *$ & $0,308 * *$ & $0,883 * *$ & & & & & \\
\hline $\mathrm{H}+\mathrm{Al}$ & 0,039 & 0,083 & $-0,201^{*}$ & 0,089 & $-0,890 * *$ & $-0,297 * *$ & $-0,644 * *$ & $-0,722 * *$ & & & & \\
\hline $\mathrm{Al}$ & 0,084 & $0,206^{*}$ & $-0,055$ & $-0,046$ & $-0,509 * *$ & $-0,215^{*}$ & $-0,425 * *$ & $-0,439 * *$ & $0,562 * *$ & & & \\
\hline S & 0,084 & $-0,066$ & $0,438^{* *}$ & $0,300^{* *}$ & $0,837 * *$ & $0,417 * *$ & $0,967 * *$ & $0,945^{* *}$ & $-0,691 * *$ & $-0,443 * *$ & & \\
\hline $\mathrm{T}$ & 0,079 & 0,053 & $0,402 * *$ & $0,610^{* *}$ & $0,304 * *$ & $0,267 * *$ & $0,497 * *$ & $0,494 * *$ & $-0,076$ & $-0,074$ & $0,495 * *$ & \\
\hline V \% & 0,040 & $-0,074$ & $0,389 * *$ & 0,130 & $0,945 * *$ & $0,380 * *$ & $0,870 * *$ & $0,924 * *$ & $-0,892 * *$ & $-0,528 * *$ & $0,908 * *$ & $0,313^{* *}$ \\
\hline
\end{tabular}

(a) $\mathrm{PRO}$ e POP = produtividade de grãos de soja e população de plantas; $\mathrm{P}, \mathrm{MO}, \mathrm{pH}, \mathrm{K}, \mathrm{Ca}, \mathrm{Mg}, \mathrm{H}+\mathrm{Al}, \mathrm{Al}, \mathrm{S}, \mathrm{T}$ e V\% são, respectivamente, o fósforo, matéria orgânica, potencial hidrogeniônico, potássio, cálcio, magnésio, acidez potencial,

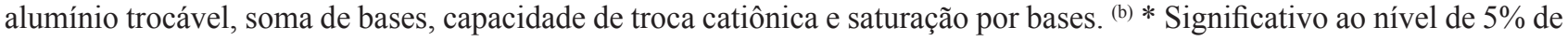
probabilidade. $* *$ Significativo ao nível de $1 \%$ de probabilidade. 


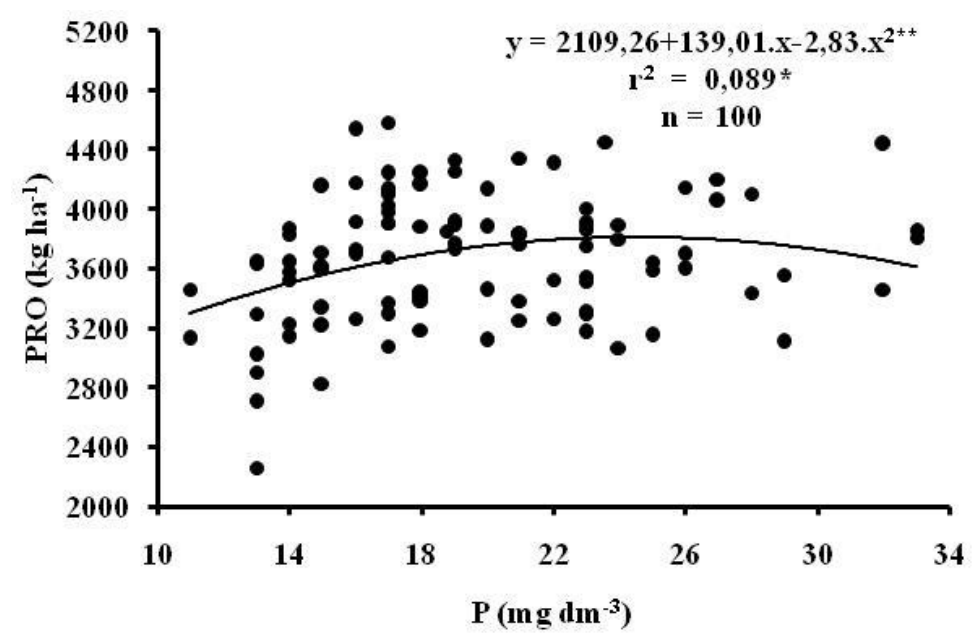

Figura 1 - Equação de regressão entre a produtividade de grãos de soja (PRO) e o teor de fósforo (P) de um Latossolo Vermelho distrófico típico. Campo Novo do Parecis (MT), 2013.

Figure 1 -Regression equation between productivity of soybeans (PRO) and the phosphorus content $(P)$ of Typic Tropudox. Campo Novo do Parecis (MT), 2013.

Assim, na Tabela 3 estão apresentados os parâmetros dos semivariogramas ajustados, podendose constatar que os atributos pesquisados, exceto a \#PRO, MO, \#Al e $\mathrm{T}$ que demonstraram efeito pepita puro, apresentaram dependência espacial. Tal fato denotou que o comportamento dessas variáveis regionalizadas que apresentaram dependência espacial não foi aleatório e que as distâncias entre pontos utilizadas na malha geoestatística foram suficientes para o estudo da variabilidade espacial. Sabe-se que uma das maneiras de avaliar o desempenho dos semivariogramas, é a sua análise a partir dos seus respectivos coeficientes de determinação espacial $\left(\mathrm{r}^{2}\right)$, de modo que o comportamento pela relação decrescente dos melhores ajustes, então foi: 1) $\mathrm{P}$ $(0,950), 2) \# \mathrm{Mg}(0,879), 3) \# \mathrm{~V} \%(0,826), 4) \# \mathrm{H}+\mathrm{Al}$ $(0,777), 5)$ \#Ca $(0,763), 6)$ \#S $(0,616), 7)$ \#pH $(0,608)$, $8) \mathrm{K}(0,398)$ e 9$)$ POP $(0,280)$.

O alcance geoestatístico é o principal parâmetro fornecido pela geoestatística, representando a distância na qual uma variável regionalizada apresenta continuidade espacial, sendo que a partir desta distância, o comportamento espacial da variável passa a ser totalmente aleatório (LEMOS FILHO et al. 2008). Pelo fato dos programas computacionais o utilizarem para monitorar o maquinário agrícola quando da aplicação de insumos com taxas variáveis (DALCHIAVON et al. 2011a), o seu conhecimento se torna essencial à agricultura de precisão. Neste contexto, seus valores ficaram entre $476,3 \mathrm{~m}$ (P) e 43,8 m (POP), o que significa que não devem ser utilizados valores inferiores a 43,8 $\mathrm{m}$ nos programas computacionais relacionados à agricultura de precisão.

Neste sentido, foi verificado que o alcance apresentou duas classes bem distintas, a primeira classe abrangendo os atributos com semivariograma gaussiano e alcance menor que 476,3 m (POP, P, \#pH, $\mathrm{K}, \# \mathrm{H}+\mathrm{Al}$, \#S e \#V\%) e a segunda classe constituída pelos atributos com semivariograma exponencial e alcance menor que $249,0 \mathrm{~m}$, sendo eles o \#Ca e o \#Mg (Tabela 3). Assim, o ajuste semivariográfico para a \#V\% ficou de acordo com o que fora observado por REICHERT et al. (2008). Para CORÁ et al. (2004), estimativas feitas com interpolação por krigagem utilizando valores de alcances maiores tendem a ser mais confiáveis por apresentarem mapas que melhor representam a realidade local. Os atributos $\mathrm{P}, \mathrm{\# Ca} \mathrm{e}$ \#Mg foram os que apresentaram os maiores valores de dependência espacial (ADE), ficando na faixa de média dependência espacial, estando de acordo com o que também fora observado por DALCHIAVON et al. (2011b) para o \#Mg e MONTANARI et al. (2014) para o P. Os demais atributos apresentaram, exclusivamente, dependência espacial baixa, variando entre $26,8 \%$ (\#S) e $31,5 \%(\mathrm{~K})$.

Para os atributos do solo, seus mapas de krigagem (Figuras 2 e 3 ) foram elaborados de acordo com as suas respectivas classes de fertilidade no solo por ser este o critério mais coerente (ZANÃO JÚNIOR et al. 2010) do que fazê-los conforme as classes sugeridas pelos programas que os geram, uma vez que as recomendações de adubação atuais 
Tabela 3 - Parâmetros dos semivariogramas e validações cruzadas para os atributos químicos em um Latossolo Vermelho distrófico típico. Campo Novo do Parecis (MT), 2013.

Table 3 - Semivariogram Parameters and cross validations for the chemical attributes of Typic Tropudox. Campo Novo do Parecis (MT), 2013.

\begin{tabular}{|c|c|c|c|c|c|c|c|c|c|c|c|}
\hline \multirow{3}{*}{ Atributo $^{(\mathrm{a})}$} & \multicolumn{11}{|c|}{ Parâmetros do ajuste } \\
\hline & \multirow{2}{*}{ Modelo $^{(b)}$} & \multirow{2}{*}{$\mathrm{C}_{\mathrm{o}}$} & \multirow{2}{*}{$\mathrm{C}_{\mathrm{o}}+\mathrm{C}$} & \multirow{2}{*}{$\begin{array}{l}\mathrm{A}_{\mathrm{o}} \\
(\mathrm{m})\end{array}$} & \multirow{2}{*}{$r^{2}$} & \multirow{2}{*}{$\mathrm{SQR}^{(\mathrm{c})}$} & \multicolumn{2}{|c|}{$\mathrm{ADE}^{(\mathrm{d})}$} & \multicolumn{3}{|c|}{ Validação cruzada } \\
\hline & & & & & & & $\%$ & Classe & $\mathrm{a}$ & $\mathrm{b}$ & $\mathrm{r}$ \\
\hline \#PRO & epp & $1,82.10^{5}$ & $1,82.10^{5}$ & - & - & - & - & - & - & - & - \\
\hline POP & gau (69) & $4,83.10^{8}$ & $6,69.10^{8}$ & 43,8 & 0,280 & $3,58 \cdot 10^{16}$ & 27,7 & BA & $8,59.10^{4}$ & 0,601 & 0,138 \\
\hline $\mathrm{P}$ & gau (429) & 9,91 & $2,15.10$ & 476,3 & 0,950 & 9,13 & 53,9 & $\mathrm{ME}$ & $4,10.10^{-1}$ & 0,978 & 0,552 \\
\hline MO & epp & 4,00 & 4,00 & - & - & - & - & - & - & - & - \\
\hline$\# \mathrm{pH}$ & gau (475) & $3,51.10^{-2}$ & $4,88 \cdot 10^{-2}$ & 93,8 & 0,608 & $4,37 \cdot 10^{-5}$ & 28,1 & BA & 0 & 0,990 & 0,356 \\
\hline $\mathrm{K}$ & gau (425) & $2,70.10^{-1}$ & $3,94 \cdot 10^{-1}$ & 93,2 & 0,398 & $7,85 \cdot 10^{-3}$ & 31,5 & BA & $4,10.10^{-1}$ & 0,778 & 0,311 \\
\hline$\# \mathrm{Ca}$ & $\exp (188)$ & 6,96 & $1,49.10$ & 222,0 & 0,763 & $1,16.10$ & 53,1 & ME & $1,00.10^{-2}$ & 0,767 & 0,348 \\
\hline$\# \mathrm{Mg}$ & $\exp (433)$ & 5,14 & $1,03.10$ & 249,0 & 0,879 & 1,36 & 50,0 & $\mathrm{ME}$ & $2,00.10^{-2}$ & 0,837 & 0,387 \\
\hline$\# \mathrm{H}+\mathrm{Al}$ & gau (523) & $2,75.10$ & $3,86.10$ & 92,2 & 0,777 & $1,39.10$ & 28,8 & BA & $-1,50.10^{-1}$ & 0,998 & 0,381 \\
\hline \#Al & epp & $1,06.10^{-1}$ & $1,06.10^{-1}$ & - & - & - & - & - & - & - & - \\
\hline$\# \mathrm{~S}$ & gau (512) & $4,58.10$ & $6,26.10$ & 95,2 & 0,616 & $8,36.10$ & 26,8 & BA & $1,80.10^{-1}$ & 0,920 & 0,341 \\
\hline $\mathrm{T}$ & epp & $3,80.10$ & $3,80.10$ & - & - & - & - & - & - & - & - \\
\hline \#V\% & gau (501) & $5,30.10$ & $7,46.10$ & 130,4 & 0,826 & $7,99.10$ & 29,0 & BA & $1,60.10^{-1}$ & 0,960 & 0,399 \\
\hline
\end{tabular}

(a) Vide Tabela 1; \# trabalhado com o resíduo do atributo; parênteses sucedendo o modelo significa o número de pares no primeiro lag; ${ }^{(b)}$ gau $=$ gaussiano, epp = efeito pepita puro, exp = exponencial; ${ }^{(c)} \mathrm{SQR}=$ soma dos quadrados dos resíduos; ${ }^{(\mathrm{d})} \mathrm{ADE}=$ avaliador da dependência espacial, sendo $\mathrm{ME}=$ média, $\mathrm{BA}=$ baixa e $\mathrm{AL}=$ alta.

são norteadas pelas classificações dos níveis dos nutrientes no solo.

No mapa da POP (Figura 2a) foi possível verificar que na maior parte da área pesquisada os valores ficaram entre 211559 e 226823 plantas ha ${ }^{-1}$, índices estes que são satisfatórios para a variedade utilizada no presente estudo, o que resultou na PRO de $3673 \mathrm{~kg} \mathrm{ha}^{-1}$, ou seja, 61 sacos, cifra bem superior à média nacional (CONAB 2014). O P apresentou teor médio a muito alto $\left(>16,0 \mathrm{mg} \mathrm{dm}^{-3}\right)$ na maior parte da área pesquisada (Figura 2b), com valores decrescendo no sentido leste-oeste, sentido este inverso ao da declividade natural do terreno, ou seja, os maiores teores de $\mathrm{P}$ foram constatados nas regiões mais baixas da área. $\mathrm{O}$ seu correto manejo no ambiente se faz de extrema importância, haja vista que, conforme salientaram SILVA et al. (2010), o P é um dos nutrientes mais limitantes à produtividade das culturas, portanto, assume grande importância na produção agrícola.

Nestas mesmas regiões de baixadas, o £pH (Figura 2c) apresentou os maiores valores $(>5,0)$, chegando próximo de 6,5 ao leste da área (região mais baixa do mapa), o que denota estar na faixa adequada à cultura da soja, uma vez que, segundo RAIJ et al. (2001), valores de pH entre 5,0 e 6,5 são considerados ideais às culturas agrícolas. Por outro lado, na região oeste foram verificados os menores valores de $\mathrm{pH}$ $(<5,0)$, logo, são os ambientes de maior acidez, representando, de certo modo, algum impedimento ao pleno desenvolvimento vegetal devido a algumas restrições de disponibilidade nutricional devido a interação dos nutrientes com o meio, influenciadas pelo $\mathrm{pH}$ do mesmo.

$\mathrm{O}$ mapa do $\mathrm{K}$ indicou que seus maiores valores ocorreram no sentido centro-leste (Figura 2d), predominando locais com teores médios $\left(>1,6 \mathrm{mmol}_{\mathrm{c}}\right.$ $\mathrm{dm}^{-3}$ ). Uma pequena porção, próxima à região central do mapa, apresentou teores de K oscilando entre 1,33$1,56 \mathrm{mmol}_{\mathrm{c}} \mathrm{dm}^{-3}$, o que caracteriza ser um ambiente de baixa fertilidade em $\mathrm{K}$.

Esse mesmo comportamento para o mapa de K foi também constatado para os mapas de $£ \mathrm{Ca}$ e $£ \mathrm{Mg}$, porém em diferentes proporções/representações 


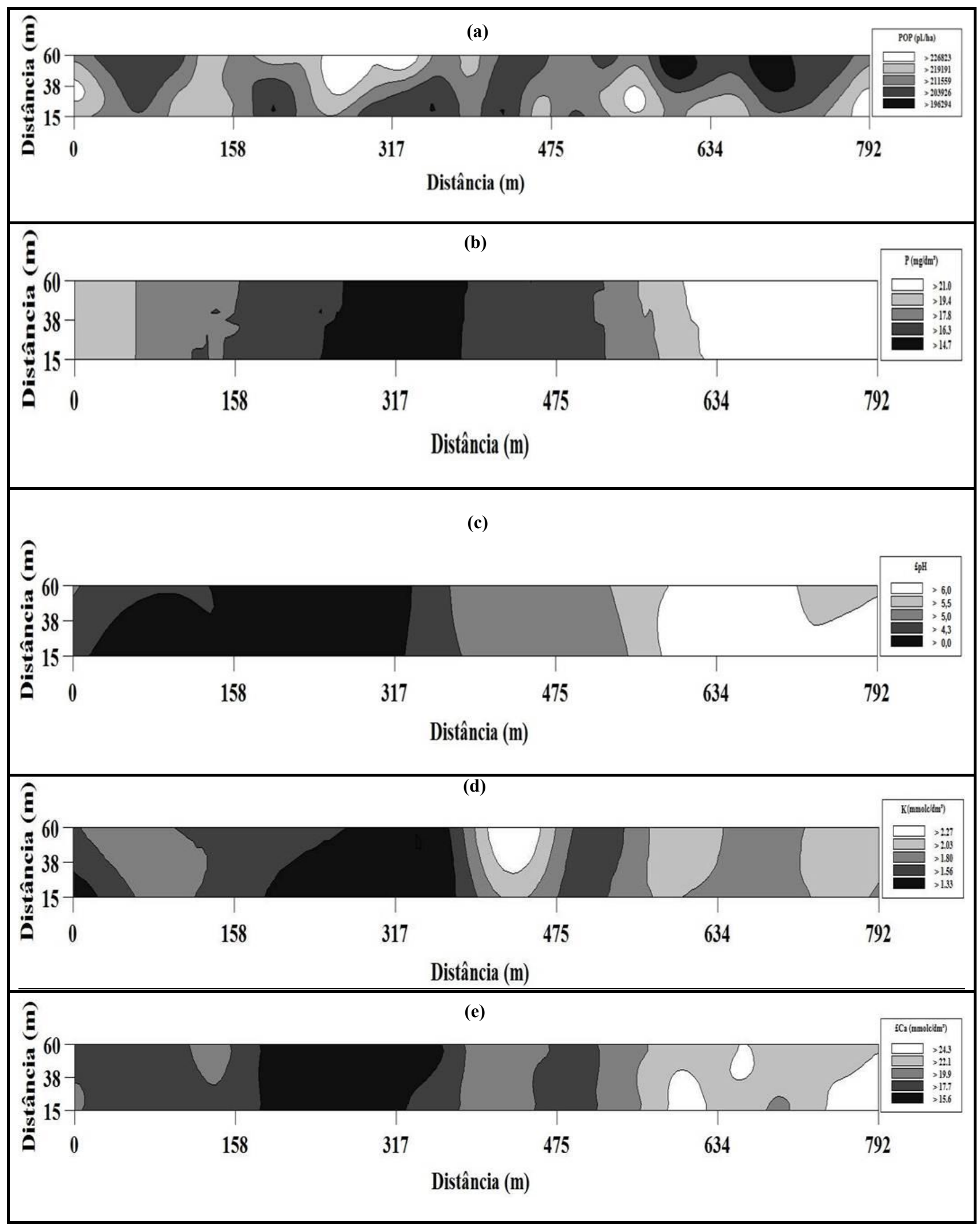

Figura 2 - Mapas de krigagem da POP e de atributos químicos (P, £pH, K e £Ca) de um Latossolo Vermelho distrófico típico. Campo Novo do Parecis (MT), 2013.

Figure 2 - Kriging maps of POP and chemical properties (P, £pH, $\mathrm{K}$ and $£ C a$ ) of Typic Tropudox. Campo Novo do Parecis (MT), 2013. 


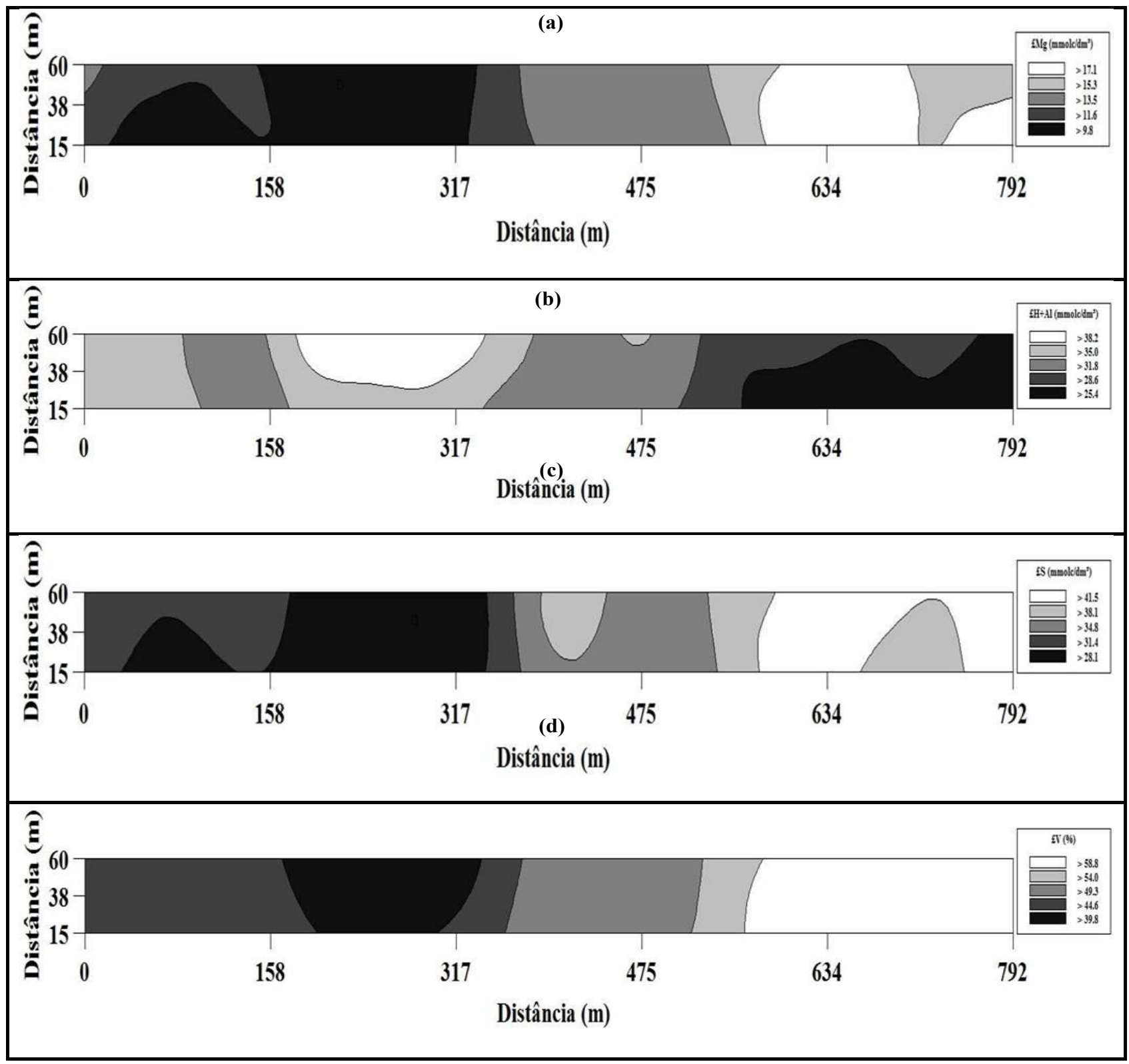

Figura 3 - Mapas de krigagem de atributos químicos (£Mg, $£ \mathrm{H}+\mathrm{Al}$, £S e $£ \mathrm{~V})$ de um Latossolo Vermelho distrófico típico. Campo Novo do Parecis (MT), 2013.

Figure 3 -Kriging maps of chemical attributes ( $£ M g, £ H+A l$, $£ S$ and $£ V$ ) of Typic Tropudox. Campo Novo do Parecis (MT), 2013.

(Figuras 2e; 3a). Contudo, tanto para o £Ca (Figura 2e) quanto para o £Mg (Figura 3a), independente do local analisado, seus teores sempre foram altos, uma vez que tal classe representa valores superiores a $>7,0$ (£Ca) e $8,0 \mathrm{mmol}_{\mathrm{c}} \mathrm{dm}^{-3}$ (£Mg). Portanto, em relação ao $\mathrm{K}, \mathrm{Ca}$ e $\mathrm{Mg}$, estes se encontravam em níveis no solo satisfatórios, possibilitando uma boa resposta da soja. Isso certamente foi reflexo do efeito residual da calagem efetuada na área em 2011, o que possibilitou a elevação dos teores das bases trocáveis em questão. Adicionalmente, é salutar destacar que grande semelhança inversa foi observada entre o mapa da $£ \mathrm{H}+\mathrm{Al}$ e os mapas das bases trocáveis anteriormente mencionados (Figuras 2 e 3), assim como entre o mapa da $£ \mathrm{H}+\mathrm{Al}$ (Figura $3 b$ ) e os mapas da $£ S$ (Figura 3c) e da $£ V$ (Figura 3d). Analogamente, semelhança, porém desta vez com relação direta, entre os mapas de $\mathrm{K}, £ \mathrm{Ca}, £ \mathrm{Mg}, £ S$ e $£ V$ também foram observadas.

Todas essas relações são amplamente discutidas na literatura, sendo de fato o comportamento esperado para a dinâmica dos solos tropicais. Assim, analisando a Figura $3 b$ e $3 c$, onde se encontram os maiores 
valores de $£ \mathrm{H}+\mathrm{Al}\left(>31,8 \mathrm{mmol}_{\mathrm{c}} \mathrm{dm}^{-3}\right)$, região centrooeste do mapa, se apresentam os menores valores de $£ \mathrm{~S}\left(<34,8 \mathrm{mmol}_{\mathrm{c}} \mathrm{dm}^{-3}\right)$. Por outro lado, os menores valores de $£ \mathrm{H}+\mathrm{Al}\left(<31,8 \mathrm{mmol}_{\mathrm{c}} \mathrm{dm}^{-3}\right)$ ocorreram nas regiões com os maiores valores de $£ \mathrm{~S}\left(>34,8 \mathrm{mmol}_{\mathrm{c}}\right.$ $\mathrm{dm}^{-3}$ ), ou seja, nas regiões centro-leste do mapa. Na Figura $3 \mathrm{~d}$, os índices da $£ \mathrm{~V}$ aparecem muito bem delineados, com índice médio (51,0-70,0\%) em mais de $50 \%$ da área, onde, na estratificação do mapa, são representados por valores $>49,3 \%$, e o restante da área com valor baixo $(26,0-50,0 \%)$, representados no mapa por valores $<49,3 \%$. Estes dados assemelhamse àqueles mencionados por CAMPOS et al. (2007) ao estudarem a dependência espacial de atributos químicos do solo em área agrícola.

Avaliando a área como um todo, foi possível constatar que houve variabilidade espacial dos atributos químicos do solo, com os valores destes atributos variando desde a classe baixa até a alta. Ambientes com acidez $(\mathrm{pH}<5,0)$ bem como saturação por bases baixa ( $\mathrm{V}<50,0 \%)$, evidenciaram a necessidade de calagem em área mínima, de maneira a homogeneizar a fertilidade do solo bem como possibilitar melhor resposta em produtividade das culturas agrícolas.

\section{CONCLUSÃO}

A maior parte dos atributos pesquisados apresentou dependência espacial, sendo possível modelar seus respectivos semivariogramas, predominantemente do tipo gaussiano.

Os alcances geoestatísticos utilizados para os atributos analisados não deverão ser inferiores a 43,8 m, por serem a distância na qual os valores dos atributos estão relacionados entre si.

$\mathrm{O}$ estudo da variabilidade dos atributos do solo bem como a adoção de técnicas que auxiliem no manejo do solo, como a geoestatística, é essencial ao adequado desenvolvimento vegetal.

\section{AGRADECIMENTOS}

Ao Grupo de Pesquisa em Fitotecnia (GPF) do Instituto Federal de Educação Ciência e Tecnologia de Mato Grosso (IFMT) - Campus Campo Novo do Parecis, pelo suporte e auxílio na condução do trabalho, e ao Programa de Iniciação Científica (PROIC) do IFMT, pelo auxílio financeiro e pela bolsa de IC concedidos.

\section{REFERÊNCIAS}

ALVES MC et al. 2009. Ambiente de produção para a canade-açúcar cultivada nas propriedades agrícolas da Usina Vale do Paraná S/A Álcool e Açúcar. Illha Solteira, 107p.

AMADO TJC et al. 2009. Atributos químicos e físicos de Latossolos e sua relação com os rendimentos de milho e feijão irrigados. Revista Brasileira de Ciência do Solo 33: 831-843.

CAIONE G et al. 2012. Produtividade e valor nutricional de variedades de cana-de-açúcar sob diferentes fontes de fósforo. Semina: Ciências Agrárias 33: 2813-2824.

CAMBARDELLA CA et al. 1994. Field-scale variability of soil properties in Central Iowa soils. Soil Science Society of American Journal 58: 1501-1511.

CAMPOS MCC et al. 2007. Dependência espacial de atributos químicos em área cultivada com cana-de-açúcar em Pereira Barreto, SP. Revista Ciência Agronômica 38: 350-359.

CARVALHO MP et al. 2003. Variabilidade espacial de atributos de um solo sob videira em Vitória Brasil (SP). Revista Brasileira de Ciência do Solo 27: 695-703.

CHAVES LHG \& FARIAS CHA. 2009. Variabilidade espacial de cobre e manganês em Argissolo sob cultivo de cana-de-açúcar. Revista Ciência Agronômica 40: 211-218. CONAB - Companhia Nacional de Abastecimento. 2014. Acompanhamento da safra brasileira: grãos, décimo segundo levantamento, abril 2014. Brasília: CONAB. 64p. CORÁ JE et al. 2004. Variabilidade espacial de atributos do solo para adoção do sistema de agricultura de precisão na cultura de cana-de-açúcar. Revista Brasileira de Ciência do Solo 28: 1013-1021.

COSTA NR et al. 2014. Produtividade de laranja correlacionada com atributos químicos do solo visando a zonas específicas de manejo. Pesquisa Agropecuária Tropical 44: 391-398.

DALCHIAVON FC et al. 2011a. Produtividade da soja e resistência mecânica à penetração do solo sob sistema plantio direto no cerrado brasileiro. Pesquisa Agropecuária Tropical 41: 8-19.

DALCHIAVON FC et al. 2011b. Variabilidade espacial da produtividade do feijoeiro correlacionada com atributos químicos de um Latossolo Vermelho Distroférrico sob sistema de semeadura direta. Bragantia 70: 908-916.

DALCHIAVON FC \& CARVALHO MP. 2012. Correlação linear e espacial dos componentes de produção e produtividade da soja. Semina: Ciências Agrárias 33: 541552.

EMBRAPA - Empresa Brasileira de Pesquisa Agropecuária. 2013. Sistema brasileiro de classificação de solos. 3.ed. Rio de Janeiro: Embrapa Solos. 353p.

FONTES LEF \& FONTES MPF. 1992. Glossário de ciência do solo. Viçosa: UFV. 142p.

GAMMA DESIGN SOFTWARE. 2004. GS+: Geostatistics for environmental sciences. 7.ed. Michigan, Plainwell: 
Gamma Desing Software. 159p.

LEMOS FILHO LCA et al. 2008. Variação espacial da densidade do solo e matéria orgânica em área cultivada com cana-de-açúcar (Saccharum officinarum L.). Revista Ciência Agronômica 39: 193-202.

MONTANARI R et al. 2014. Produção de cana-de-açúcar correlacionada com o teor de fósforo em Mato Grosso do Sul. Revista de Agricultura Neotropical 1: 1-9.

PIMENTEL-GOMES F \& GARCIA CH. 2002. Estatística aplicada a experimentos agronômicos e florestais: exposição com exemplos e orientações para uso de aplicativos. 11.ed. Piracicaba: FEALQ. 309p.

RAIJ BV et al. 2001. Análise química para avaliação da fertilidade de solos tropicais. Campinas: Instituto Agronômico. 285p.

RAIJ BV et al. 1997. Recomendação de adubação e calagem para o Estado de São Paulo. 2.ed. Campinas: IAC. 285p. (Boletim Técnico 100).

REICHERT JM et al. 2008. Variabilidade espacial de Planossolo e produtividade de soja em várzea sistematizada: análise geoestatística e análise de regressão. Ciência Rural 38: 981-988.

SCHLOTZHAVER SD \& LITTELL RC. 1997. SAS System for elementary statistical analysis. 2.ed. Cary: SAS. 905p.

SEIDEL EJ \& OLIVEIRA MS. 2014. Novo índice geoestatístico para a mensuração da dependência espacial. Revista Brasileira de Ciência do Solo 38: 699-705.

SHAPIRO SS \& WILK MB. 1965. An analysis of variance test for normality (complete samples). Biometrika 52: 591611.

SILVA SA et al. 2010. Variabilidade espacial do fósforo e das frações granulométricas de um Latossolo Vermelho Amarelo. Revista Ciência Agronômica 41: 1-8.

SOUZA ZM et al. 2014. Número de amostras na análise geoestatística e na krigagem de mapas de atributos do solo. Ciência Rural 44: 261-268.

ZANÃO JÚNIOR LA et al. 2010. Variabilidade espacial de atributos químicos em diferentes profundidades em um Latossolo em sistema de plantio direto. Revista Ceres 57: 429-438.

ZANÃO JÚNIOR LA et al. 2007. Variabilidade espacial do $\mathrm{pH}$, teores de matéria orgânica e micronutrientes em profundidades de amostragem num Latossolo Vermelho sob semeadura direta. Ciência Rural 37: 1000-1007. 